\title{
Endomyocardial Fibrosis: Echocardiographic Profile
}

\author{
Ramachandran Muthiah \\ Thoothukudi Medical College Hospital, Thoothukudi, India \\ Email: cardioramachandran@yahoo.co.uk
}

Received 13 April 2016; accepted 24 May 2016; published 27 May 2016

Copyright (C) 2016 by author and Scientific Research Publishing Inc.

This work is licensed under the Creative Commons Attribution International License (CC BY).

http://creativecommons.org/licenses/by/4.0/

(c) (i) Open Access

\begin{abstract}
Evolution of two-dimensional and color Doppler echocardiography identified the typical features of Endomyocardial Fibrosis (EMF) such as dense ventricular apical fibrosis, dysfunction of atrioventricular valve and sub-valvular apparatus and cavity dimensions. This is the tool used most for the diagnosis of EMF in areas where the disease is endemic in Africa. Today echocardiography is used as a screening tool to identify cases of EMF at the community level and it could be confirmed at the bed side. Background of these cases highlighted the echocardiographic features of EMF in different age groups and the oldest one reported at the age of 85 years in a female in advanced stage at this coastal district of Thoothukudi in India.
\end{abstract}

\section{Keywords}

Apical Fibrosis, African Heart, Pericardial Effusion, EMF Syndrome, Mushroom Sign

\section{Introduction}

Endomyocardial Fibrosis (EMF) is an idiopathic disorder of the tropical and sub-tropical regions of the world that is characterized by the development of restrictive cardiomyopathy [1]. In EMF, the underlying process produces patchy fibrosis of the endocardial surface of the heart. Fibrosis increases the stiffness of the heart, resulting in the restrictive physiology. Ventricular stiffness along with atrioventricular (AV) valvular regurgitation results in atrial enlargement, which has been linked to atrial arrhythmias such as atrial fibrillation. Fibrosis also reduces conduction velocity, impairs activation pattern and may provide the substrate for wave breaks and reentry [2]. Atrial fibrillation has been reported in more than $30 \%$ of patients with EMF followed by other rhythm or conduction abnormalities like junctional rhythm, heart blocks, and intraventricular conduction delay [3].

EMF is said to be the most common form of heart disease in Uganda hospitals, where it accounts for nearly $20 \%$ of cases referred to an echocardiographic service [4]. The only epidemiological survey performed in Africa 
was based on an echocardiographic diagnosis of EMF in the Inharrine district of Mozambique. In a sample of 948 inhabitants from an endemic area and aged between 4 and 45 years old, a prevalence of 8.9\% was reported, suggesting that EMF was a major form of heart disease in this region. Transthoracic echocardiography can be used to assess the severity and pathologic abnormalities of EMF [5]. In a series of patients from Mozambique, echocardiographic description coincided with the intraoperative findings in more than $80 \%$ of patients, the concordance being absolute for the most important pathological lesions of EMF, namely fibrosis of the posterior papillary muscle and leaflets of the wall, left ventricular apical fibrosis, thickening of the atrioventricular valve leaflets, right ventricular obliteration, retraction and ventricular thrombi.

The typical mushroom like appearance of apical fibrosis in ventricles in echocardiography and the oldest one Right ventricular (RV) EMF detected in a female at the age of 85 years were the salient features described and so it had been reported.

\section{Case Reports}

\section{Case 1 (Right ventricular EMF in a female aged 54 years)}

A 54 years old woman presented with puffiness of face and bilateral pedal edema of one month duration. Blood chemistry revealed normal. ECG showed Twave inversions in right precordial leads. X-ray chest revealed normal. Transthoracic 2D echocardiographic images revealed RV apical fibrosis with an appearance of mushroom in apical four chamber view as shown in Figure 1 and parasternal long axis view as shown in Figure 2.

Case 2 (Right ventricular EMF in a male aged 47 years)

The clinical features are similar to case-1. Transthoracic 2D echocardiographic imaging showing RV apical fibrosis with mild tricuspid regurgitation and a dilated atria as shown in Figure 3.

Case 3 (85 years old female with advanced RV EMF)

Clinical features are similar to case 1 and in addition, the patient had conduction disturbances such as heart block (III ${ }^{\text {rd }}$ degree). 2D echocardiographic imaging revealed RV apical fibrosis with a thick, calcified moderator band engulfed in fibrosis, causing ventricular wall retraction and resulting an apical notch in the RV wall, giving an appearance of the map of Africa suggesting the advanced stage of the RV EMF as shown in Figure 4.

Case 4 (Left ventricular (LV) EMF in a 10 year old boy)

Clinical features are similar to case 1 and masquerading as acute nephritis and nephritic syndrome, idiopathic dilated cardiomyopathy, rheumatic heart disease, but auscultation of the heart and renal parameters were normal. 2D echocardiographic imaging revealed LV apical obliteration with fibrosis and a dilated left ventricle suggesting a subacute stage of LV EMF as shown in Figure 5.

Case 5 (Left ventricular EMF in an adult aged 54 years)

The clinical features are similar to case 1 and 2D echocardiographic imaging showing the dense fibrosis in LV apex and a dilated left ventricle with an ejection fraction of 50\% as shown in Figure 6.

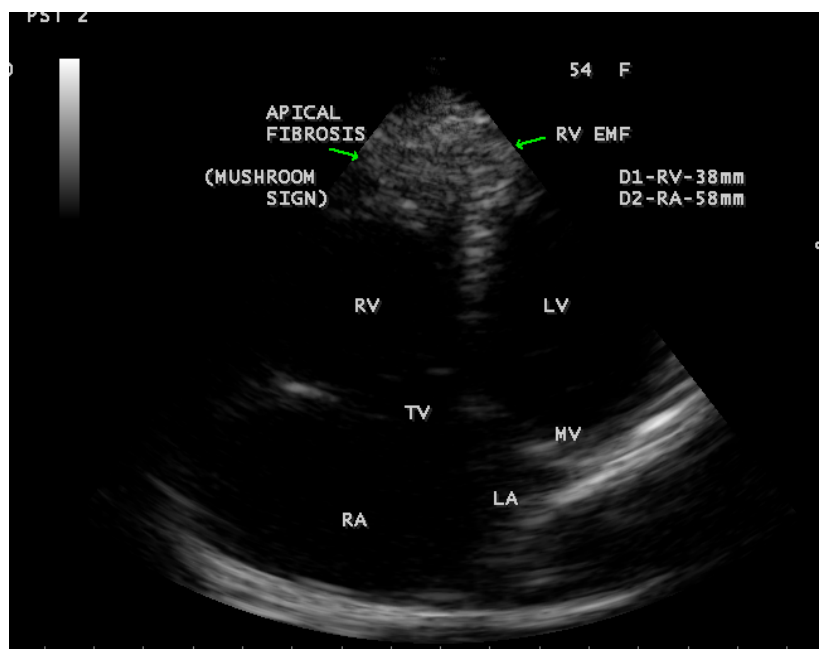

Figure 1. Apical 4 chamber view showing RV apical fibrosis with an appearance of a mushroom. 


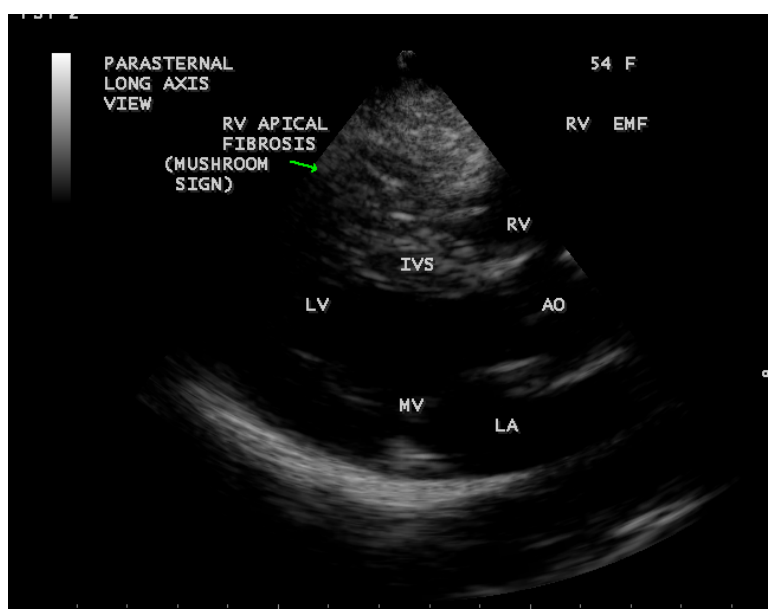

Figure 2. Parasternal long axis view showing a dense RV apical fibrosis.

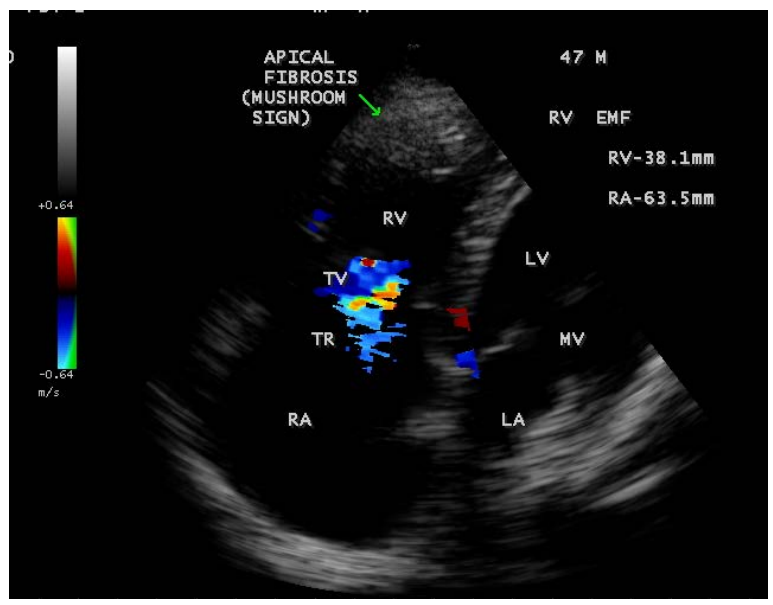

Figure 3. Apical 4 chamber view showing RV apical fibrosis, mild tricuspid regurgitation and a dilated atria.

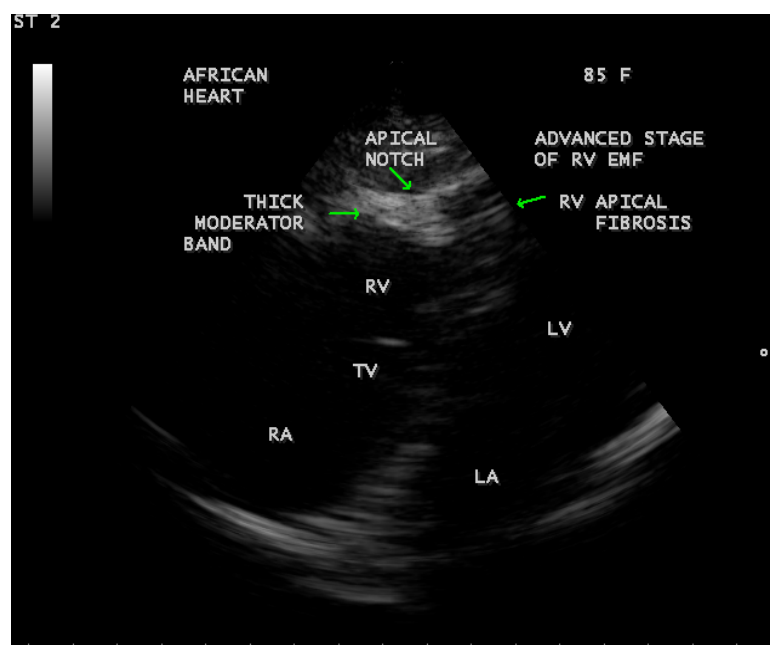

Figure 4. Tilted apical four chamber view showing RV apical fibrosis, thick and calcified moderator band, apical notch on the RV wall simulating the description as "African heart”. 


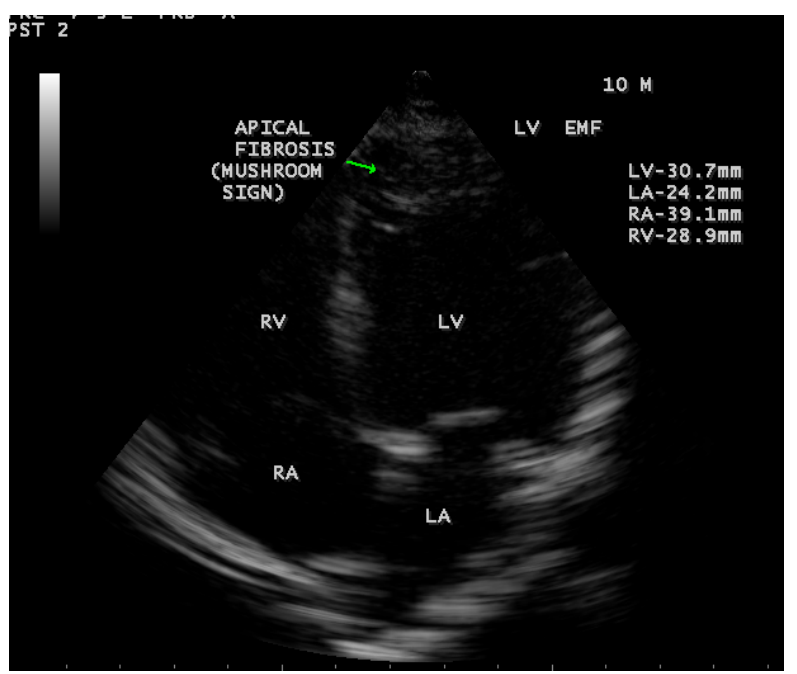

Figure 5. This figure shows LV apical fibrosis, dilated left ventricle and normal left atrium.

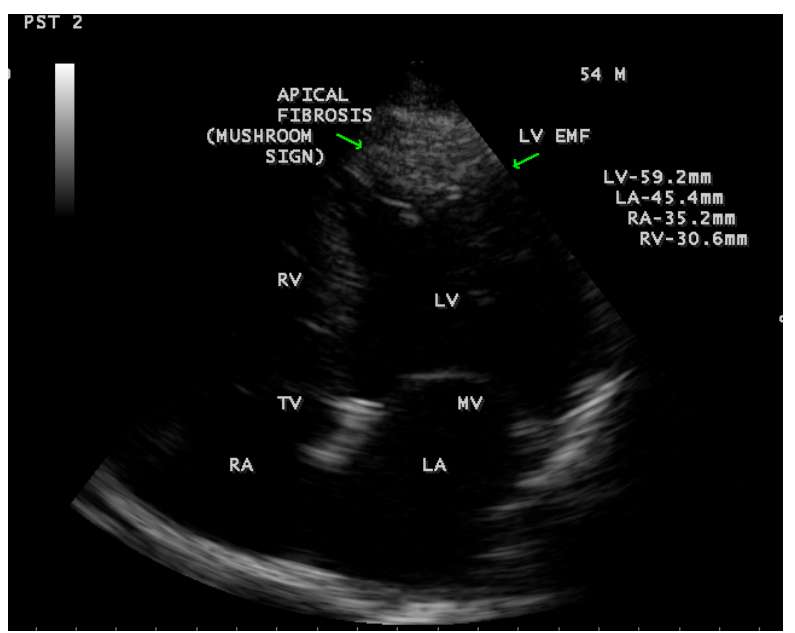

Figure 6. This figure shows the LV apical fibrosis with dilated cardiac chambers in LV EMF.

\section{Case 6 (Left ventricular EMF with pericardial effusion in a female aged 23 years)}

23 years old female presented with puffiness of face and chest discomfort during breathing with a history of 2 to 3 episodes of febrile illness during childhood. Echocardiographic screening revealed Left ventricular EMF with mild pericardial effusion as shown in Figure 7 and typical mushroom sign of apical fibrosis in RV EMF in a 30-year old female as shown in Figure 8, in Biventricular EMF in a 8-year old boy as shown in Figure 9. Screening of family members revealed normal.

These cases are responded to diuretics and improvement in their life style occurred and they are advised periodic follow up. There is no in-hospital mortality so far among EMF cases detected and now flourishing as two to three cases monthly in this endemic area of Thoothukidi similar to the endemic rheumatic heart diseases in this region. It is a coastal district with hot and humid climatic conditions and majority are having a history of febrile illness years back before the onset this illness. Screening of family members revealed no abnormalities and so it is not familial.

\section{Discussion}

\subsection{Echocardiographic Features}

The wide spectrum of distribution and severity of the fibrotic lesions, as well as the changes in heart shape and 


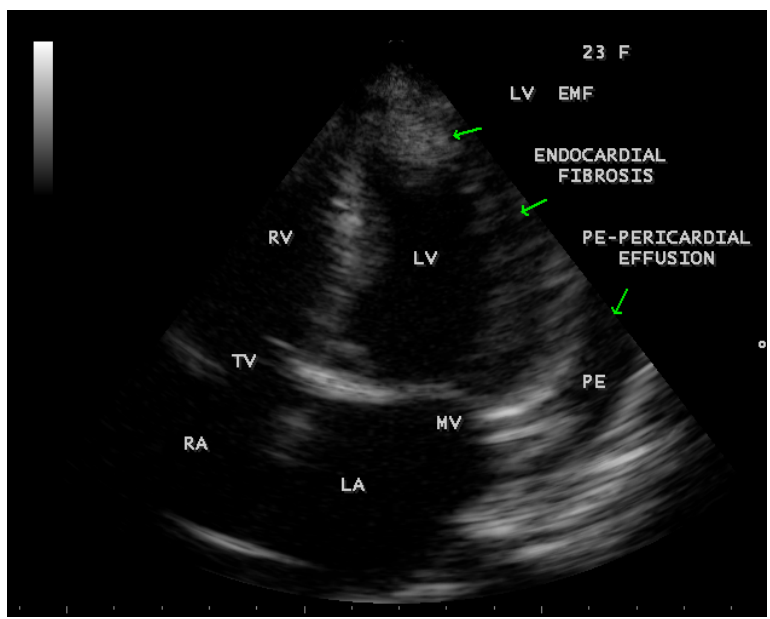

Figure 7. This figure shows endocardial fibrosis on left ventricular wall with mild pericardial effusion.

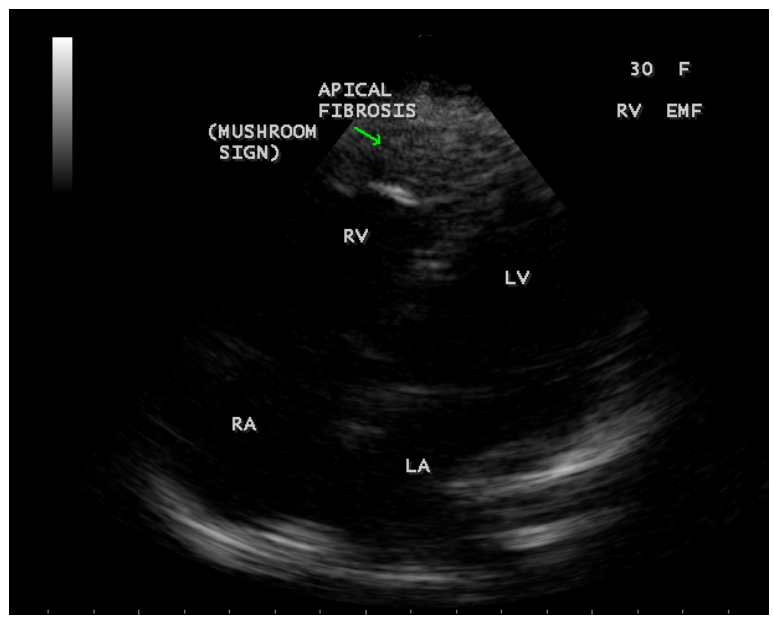

Figure 8. Apical four chamber view showing RV EMF in a 30-year-old female.

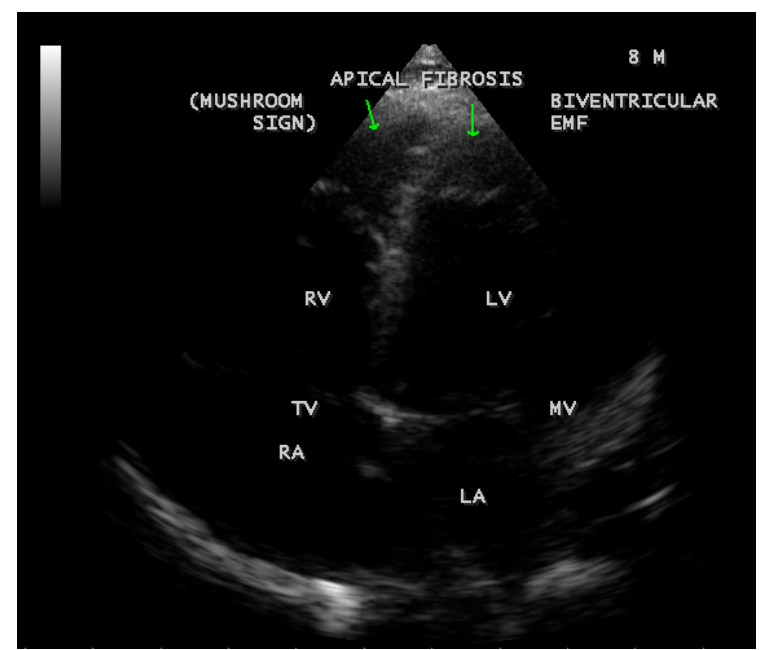

Figure 9. This figure shows the mushroom sign in a 8-year old boy with Biventricular EMF. 
distortion mandate a careful and comprehensive echocardiographic evaluation of each patient, using the usual and less conventional views. The hallmark of the established EMF is the presence of thickened endocardium, ventricular obliteration and dilated atria. Less specific echocardiographic abnormalities include diffuse AV valve leaflet thickening, enhanced echodensity of the moderator band or trabeculae, the presence of thickened left ventricular false tendon [6].

Thickening of the endocardium is the most characteristic feature of established EMF [7] [8]. Echocardiography can detect the presence of a thickened endocardium on the ventricular wall as shown in Figure 7. The most striking and consistent features are increased amplitude echoes at the right ventricular trabecular region, left ventricular apex and the region of posterior mitral leaflet [9]. Ventricular thrombi are frequently seen in normally contracting ventricle in early stages of EMF, as a part of the initial process that leads to endocardial fibrosis [10].

\subsection{Ventricular Apical Fibrosis (Mushroom Sign)}

Obliteration of the apex of the ventricles by fibrosis is the characteristic feature of EMF. In right ventricular EMF, the trabecular portion of the ventricle is separated from the remaining cavity by a large fibrotic endocardial plaque, underneath which there is myocardium of apparently normal texture [11]. This large fibrotic plaque obliterating the apex of the ventricle giving a typical appearance of mushroom and designated as "mushroom sign" which is a classical feature of EMF in echocardiography as shown in Figures 1-3, Figure 5, Figure 6 and Figure 8. The mechanism of right ventricular cavity obliteration corresponds to compaction of trabeculae between the thickened fibrous endocardium and the epicardium, excluding the trabecular portion of the ventricle from the circulation [12]. The fibrotic process produces an "artificial floor" to the right ventricle, separating the inflow cavity from the obliterated muscular trabecular part, which does not contain thrombus or blood. The moderator band is lost, engulfed in the endocardial fibrosis as shown in Figure 4. The progressive fusion of trabeculae between the thickened endocardium and epicardium results in retraction of the ventricular cavity. There is a reduction in the distance between the epicardium and the false floor and this process is associated with pulling of the wall by the retracted tricuspid valve apparatus, resulting in the distinctive finding of advanced rightsided EMF called "apical notch" as shown in Figure 6. The "apical notch" gives the heart a shape that resembles the map of Africa, hence the designation "Heart of Africa" [13].

Left ventricular obliteration affects both the apex and recesses of the posterior mitral leaflet excluding these parts from the ventricular cavity. It is thought that obliteration by thrombi and subsequent scarring and fibrosis are the mechanisms involved, both leading to reduction of the diastolic properties of the ventricles. Also, thrombi may involve the sub-valvular apparatus, leading to scarring and fibrosis of the leaflets to the ventricular wall, therefore resulting in leaflet movement restriction and AV valve dysfunction. Left ventricular apex is never retracted, it becomes thicker leading to considerable reduction of the longitudinal diameter of the ventricle, resulting in a spherical ventricular cavity as shown in Figure 5. The fibrotic process of EMF involves the atrioventricular (AV) valve and sub-valvular apparatus and masquerading as rheumatic heart disease in endemic areas for both diseases. In chronic rheumatic heart disease, leaflet thickening is usually restricted to the tip of the valve, extends to the chordae, and is not associated with obliteration of the ventricle [14] [15]. Both the restriction to the ventricular filling and the $\mathrm{AV}$ valve regurgitation results in increase in atrial pressure, leading to progressive atrial dilatation. Outflow tracts of the ventricles and semilunar valves are spared from the disease process of EMF.

\subsection{Echocardiographic Criteria}

Mocumbi et al. have described a set of criteria for the diagnosis and classification of endomyocardial fibrosis as shown in Table 1 and this can apply only to the disease in the advanced stage.

A definite diagnosis of EMF was made in the presence of two major criteria or one major criterion associated with two minor criteria.

\subsection{Clinical Features}

The clinical features of EMF depend on the stage of disease and the anatomic involvement of the heart. 30\% to $50 \%$ of children and adolescents report as initial illness with fever, chills, night sweats, facial swelling and 
Table 1. Criteria for diagnosis and assessment of the severity of Endomyocardial fibrosis.

\begin{tabular}{ll}
\hline Criterion & Score \\
\hline Major criteria & 2 \\
Endomyocardial plaques $>2$ mm in thickness & 3 \\
Thin ( $\leq 1 \mathrm{~mm}$ ) endomyocardial patches affecting more than one ventricular wall & 4 \\
Obliteration of right ventricular or left ventricular apex & 4 \\
Thrombi or spontaneous echo contrast without severe ventricular dysfunction & 4 \\
Retraction of the right ventricular apex (right ventricular apical notch) & \\
Atrioventricular-valve dysfunction due to adhesion of the valvular apparatus to the \\
ventricular wall (The score is assigned according to the severity atrioventricular \\
regurgitation) \\
$\begin{array}{l}\text { Minor criteria } \\
\text { Thin endomyocardial patches localized to one ventricular wall }\end{array}$ \\
$\begin{array}{l}\text { Restrictive flow pattern across mitral or tricuspid valves } \\
\text { Pulmonary-valve diastolic opening }\end{array}$ \\
$\begin{array}{l}\text { Diffuse thickening of the anterior mitral leaflet } \\
\text { Enlarged atrium with normal-size ventricle }\end{array}$ \\
M-movement of the interventricular septum and flat posterior wall \\
Enhanced density of the moderator or other intraventricular bands \\
\hline
\end{tabular}

A total score of $<8$ = mild EMF; 8 to 15 = moderate EMF; $>15$ = severe EMF.

urticaria [16]. This illness may disappear, or it may leads to rapidly developing cardiac failure or it may evolve into established and apparently inactive EMF with predominant right ventricular or left ventricular disease. The exudative pericardial effusion of variable degree is a common presentation as illustrated in Figure 7. These observations suggest that EMF is a systemic inflammatory disease, which has been called the "EMF syndrome" [17].

\section{Conclusion}

Right and Left ventricular Endomyocardial Fibrosis in different age groups have been described by Transthoracic 2D Echocardiographic imaging. A right ventricular EMF at the age of 85 years in an elderly woman and a Left ventricular EMF with mild pericardial effusion at the age of 23 years in a young woman were detected in this coastal district of Thoothukudi in India.

\section{References}

[1] Dato, I. (2014) How to Recognize Endomyocardial Fibrosis? Journal of Cardiovascular Medicine (Hagerstown).

[2] Rossi, S., Baruffi, S., Bertuzzi, A., Miragoli, M., Corradi, D., Maestri, R., et al. (2008) Ventricular Activation Is Impaired in Aged Rat Hearts. American Journal of Physiology, Heart and Circulatory Physiology, 295, H2336-H2347. http://dx.doi.org/10.1152/ajpheart.00517.2008

[3] Gupta, P.N., Valiathan, M.S., Balakrishnan, K.G., et al. (1989) Clinical Course of Endomyocardial Fibrosis. British Heart Journal, 62, 450-454.

[4] Freers, L., Mavania-Kizza, M., Ziegler, H. and Rutaceinnio, M. (1996) Echocardiographic Dignosis of Heart Disease in Uganda. Tropical Doctor Journal, 26, 125-128.

[5] Mocumbi, A.O., Carrilho, C., Sarathchandra, P., Ferreira, M.B., Yacoub, M.H. and Burke, M. (2010) Echocardiography Accurately Assesses the Pathological abnormalities of Chronic Endomyocardial Fibrosis. International Journal of Cardiovscular Imaging, 6, 445-452.

[6] Mocumbi, A.O., Ferreiro, M.B., Sidi, D. and Yacoub, M.H. (2008) A Population Study of Endomyocardial Fibrosis in a Rural Area of Mozambique. New England Journal of Medicine, 369, 43-49. http://dx.doi.org/10.1056/NEJMoa0708629

[7] Okereke, O.U.J., Chikwendu, V.C., Ihenacho. H.N.C. and Ikeh, V.O. (1991) Non-Invasive Diagnosis of Endomyocardial Fibrosis in Nigeria Using Two-Dimensional Echocardiography. Tropical Cardiology, 17, 97-103. 
[8] Connor, D.H., Somers, K., Hutt, N.S.R., Manion, W.C. and D’Arbela, P.G.D. (1967) Endomyocardial Fibrosis in Uganda (Davies’ Disease). Part I. American Heart Journal, 74, 687-709. http://dx.doi.org/10.1016/0002-8703(67)90509-1

[9] Vijayaraghavan, G., Davies, J., Sadanandan, S., Spry, C.J.F., Gibson, D.G. and Goodwin, J.F. (1983) Echocardiographic Features of Tropical Endomyocardial Disease in South India. British Heart Journal, 50, 450-459. http://dx.doi.org/10.1136/hrt.50.5.450

[10] Berenstein, C.S., Pinero, G., Marcotegui, M., Brunoldi, R., Blanco, M.V. and Lerman, J. (2000) Usefulness of Echocardiography and Doppler Echocardiography in Endomyocardial Fibrosis. Journal of American Society of Echocardiography, 13, 226-230.

[11] Trigo, J., Camacho, A., Gago, P., Candeias, R., Santos, W., Marques, N., Matos, P., Brandao, V. and Gomes, V. (2010) Endomyocardial Fibrosis. Revista Portuguesa de Cardiologia, 29, 445-449.

[12] Mocumbi, A.O., Sidi, D., Vouhe, P. and Yacoub, M. (2007) An Innovative Technique for The Relief of Right Ventricular Trabecular Cavity Obliteration in Endomyocardial Fibrosis. Journal of Thoracic Cardiovascular Surgery, 134, 1070-1072. http://dx.doi.org/10.1016/j.jtcvs.2007.04.062

[13] Davies, J.N.P. (1960) Some Considerations Regarding Obscure Diseases Affecting the Mural Endocardium. American Heart Journal, 19, 600-630. http://dx.doi.org/10.1016/0002-8703(60)90464-6

[14] Saraiva, L.R., Carneiro, R.W., Arruda, M.B., Brindeiro Filho, D. and Lira, V. (1999) Mitral Valve Disease with Rheumatic Appearance in the Presence of Left Ventricular Endomyocardial Fibrosis. Arquivos Brasileiros de Cardiologia, 72, 327-332. http://dx.doi.org/10.1590/S0066-782X1999000300006

[15] Metras, D., Quezzin, Coulibaly, A., Quattara, K., Bertrand, E. and Chauvet, J. (1983) Endomyocardial Fibrosis Masquerading as Rheumatic Mitral Incompetence, A Report of Six Surgical Cases. Journal of Thoracic Cardiovascular Surgery, 86, 753-756.

[16] Freers, I., Hakim, J., MyanjaKizza, H. and Parry, F. (2004) The Heart, Endomyocardial Fibrosis, Still a Mystery after 60 Years. Principles of Medicine in Africa, 837-886.

[17] Freers, J., Masembe, V., Schmauz, R. and Mayanja, H., (2000) Endomyocardial Fibrosis (EMF) Syndrome in Uganda. The Lancet, 355, 1994-1995. http://dx.doi.org/10.1016/S0140-6736(05)72932-9 